Témoigner Témoigner. Entre histoire et mémoire

Getuigen Revue pluridisciplinaire de la Fondation Auschwitz

$124 \mid 2017$

La musique dans les camps

\title{
Hélios Azoulay « Sauvée des cendres »
}

'Sauvée des cendres'. Interview met Hélios Azoulay

\section{Daniel Weyssow}

\section{Q OpenEdition}

\section{Journals}

\section{Édition électronique}

URL : https://journals.openedition.org/temoigner/5774

DOI : 10.4000/temoigner. 5774

ISSN : 2506-6390

\section{Éditeur :}

Éditions du Centre d'études et de documentation Mémoire d'Auschwitz, Éditions Kimé

\section{Édition imprimée}

Date de publication : 2 avril 2017

Pagination : 124-134

ISBN : 978-2-930953-00-7

ISSN : 2031-4183

Référence électronique

Daniel Weyssow, « Hélios Azoulay « Sauvée des cendres » », Témoigner. Entre histoire et mémoire [En ligne], 124 | 2017, mis en ligne le 30 novembre 2021, consulté le 01 décembre 2021. URL : http:// journals.openedition.org/temoigner/5774 ; DOI : https://doi.org/10.4000/temoigner.5774 


\section{Hélios Azoulay}

\section{«Sauvée des cendres »} $\rightarrow$ Daniel Weyssow
(Fondation Auschwitz)

Le dernier CD d'Hélios Azoulay et l'Ensemble de Musique Incidentale intitulé Sauvée des cendres se compose principalement d'un opéra inachevé de Victor Ullmann, Le 30 mai 1431, tiré du récit de Jeanne d'Arc qui fut, comme on le sait, brûlée vive par l'Inquisition. Concue dans le ghetto de Theresienstadt, cette œuvre d'Ullmann s'avérait plus que prémonitoire. En toute liberté, Hélios Azoulay a relevéle défi de la développer musicalement. À l'image du Big Bang, en partant des rudiments des deux seules pages écrites de la partition. Àlarrivée, une création croisée Ullmann-Azoulay / Azoulay-Ullmann dign d'une musique des sphères, où la partition, ainsi relevée et achevée, exprime par le timbre le plus juste l'atroce destinée des victimes de la arbere. Entretie (3) (3 décembre 2016 àla rondation Auschwitz (Bruxelles)

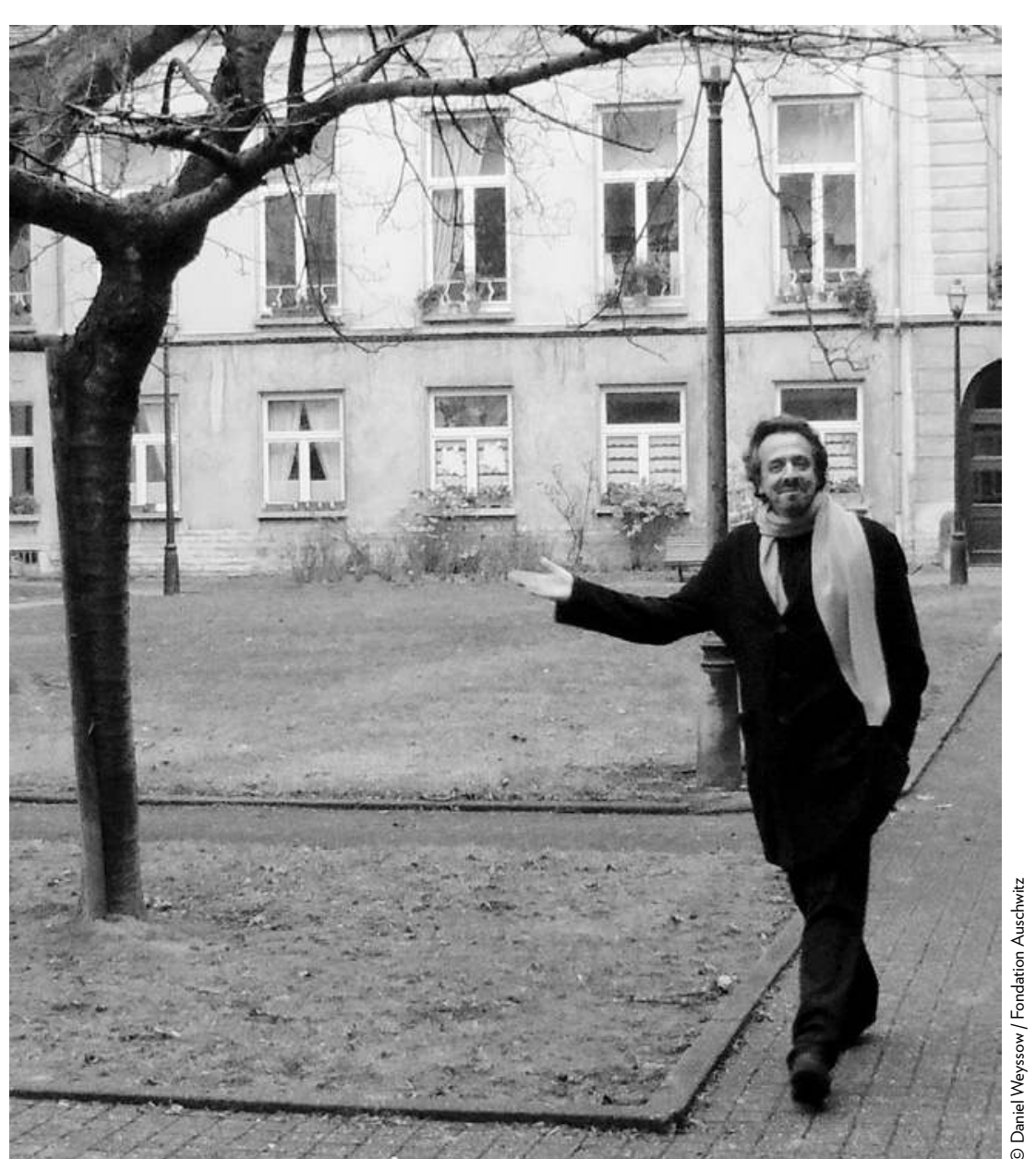

Hélios Azoulay, nous sommes très heureux de vous revoir' afin d'évoquer la sortie de votre dernier opus intitulé « "Sauvée des cendres », créé avec vos musiciens de l'Ensemble de Musique Incidentale : Marielle Rubens, Pablo Schatzman, Teona Kharadze, Baptiste Vay et Dimitri Maslennikov.

Votre précédent $C D$ «.... même à Auschwitz » était déjà une anthologie des musiques composées dans les camps de concentration nazis. On y trouvait des compositions d'Émile Goué, Germaine Tillion, Hans Krása, Ilse Weber, Robert Dauber, Karel Berman, Gideon Klein, une devous-mâme, $n^{\circ} 78707$, d'après le journa linédit d'un déporté (1) a Buchenwald. Et votre disque se concluait par lesquisse d'une ouverture d'un opéra
inachevé, Le 30 mai 1431. inachevé, Le 30 mai 1431.

Ce fragment, signé Viktor Ullmann, est le coeur de «Sauvée des cendres ». Mais l'album comprend également une magnifique berceuse d'Aleksander Volkoviski issue du ghetto de Wilno, une autre d'Aaron Liebeskind, une mélodie d'llse Weber, ainsi qu'un quatuor à cordes dont vous êtes l'auteur, dédié à Violette Jacquet-Silberstein, violoniste dans l'orchestre des femmes d'Auschwitz.

Mais tout d'abord, pourquoi «Sauvée des cendres»?

Jeanne d'Arc sauvée des cendres était au départ le titre trouvé assez instinctivement - mais pas sans évidentes résonances - du projet de financement participatif qui a permis au disque d'exister. Ce financement a été, du reste, un succès absolument dingue. Énormément de presse, un soutien sublime de tant et tant d'anonymes, d'amis, d'artistes, et même un membre de l'Académie Française... d'anonymes, d'amis, d'artistes, et même un membre de l'Académie Française...
Mais une seule institution : la vôtre! La Fondation Auschwitz. Du reste votre parMais une seule institution : la vôtre ! La Fondation Auschwitz. Du reste votre par-
ticipation est pour moi un symbole très fort. Quelques heures après les attentats ticipation est pour moi un symbole très fort. Quelques heures après les attentats
effrayants dans le métro bruxellois, vous avez décidé de combler, à l'euro près, la somme qui manquait pour arriver aux 15000 euros nécessaires. Je ne crois pas que l'on pouvait faire plus juste et plus fort pour clamer à cette heure précise, et si tragique, l'engagement que nous devons tous avoir pour l'Art et ce qu'il représente comme élan de vie ! J’en étais bouleversé. Et le suis encore.

Mais revenons à ce chef d'œuvre inachevé de Viktor Ullmann, Le 30 Mai 1431. Écrit à Theresienstadt, il porte certainement à l'un de ses apogées le récit de Jeanne d'Arc. Pouvez-vous, avant tout, nous dire un mot sur la vie et l'œuvre de Viktor Ullmann?

Viktor Ullmann est un compositeur que l'on joue aujourd'hui - et c'est tant mieux - de plus en plus. On prend conscience enfin de sa place tout à fait particulière dans l'histoire de la musique du XX $\mathrm{X}^{\mathrm{e}}$ siècle. Et s'il n’avait achevé la fin de sa course, la course de la vie, comme la course de la création, dans la chambre à gaz d'Auschwitz, il aurait été sans aucun doute un compositeur « qui compte». Il a été lélève d'Arnold Schönberg mais son langage n'est pas du tout strictement schönberguien. Il possède une singulière et puissante force expressionniste. Il était né dans une famille qui s'était convertie au catholicisme. Mais si la religion ne l'intéressait guère, c’était un être d'une grande spiritualité. Il était anthroposophe... L'anthroposophie étant une
(1) Daniel Weyssow, « Entretien avec Hélios Azoulay, in

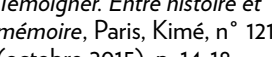


espèce de mysticisme créé par un certain Rudolf Steiner, pour lequel d’ailleurs, un moment, il a une espèce de dévotion incroyable. Il a tenu un temps la librairie du mouvement à Stuttgart et arrêté la musique. Mais il s'y est remis avant d'aboutir au ghetto de Theresienstadt et durant le temps qu'il y passa, il nous a laissé des partitions d'une grande maturité, dont son opéra le plus connu, Der Kaiser von Atlantis [l'Empereur d'Atlantis], son œuvre la plus jouée, peut-être... Cependant, de nombreuses ouvres de Viktor Ullmann ont été composées à Theresienstadt.

Et c'est là, noyé par la frayeur des convois qui partent vers l'est, d'où personne - Etcest là noù personne ne revient, quen octobre 1944, il confie à un ami, qui va survivre, une valise contenant toutes les œuvres composées a Theresienstadt, certaines d'inspiration juive et hébraïque, avec des mélodies, des chœurs, etc. Des œuvres qui nous interrogent dont l'ouverture d'un opéra intitulé Don Quichotte danse le Fandango. Du reste, c'est à l'intérieur de cette ouverture d'opéra qui n'a pas vu le jour que se trouven les deux pages pour piano du 30 mai 1431. C'est la date à laquelle Jeanne d'Arc périt sur le bûcher à Rouen. Mais au-delà des deux pages, enfin avec les deux pages, il y a également deux actes en vers rimés achevés, complétés, que j’ai été le premier à traduire et à publier dans mon dernier livre, L'enfer aussi a son orchestre.

\section{Ces deux actes se trouvaient également dans le Don Quichotte?}

Je pense que c'était à part. Mais en tout cas, quand on sait que Cervantes est un juif marrane, il y a quelque chose d'hallucinant dans cette histoire : un Juif converti au catholicisme, marié malgré tout à une femme juive, devenant anthroposophe, revenant finalement vers le judaïsme à Theresienstadt, et qui prend Cervantes, un marrane, pour «abriter» Jeanne d'Arc !

En effet. Donc, de cette œuvre, Le 30 mai 1431, Ullmann en aura rédigé et achevê le livret, mais pas la musique dont vous avez retrouvé les deux seules pages écrites de la partition. On peut être au premier abord surpris de constater que le personnage de

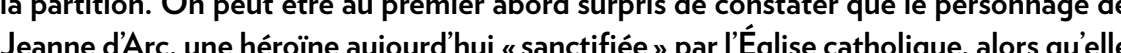
Jen lavait exécutée en la brûlant vive, soit également devenue un emblème au sein de la communauté juive. C'est une question que Pierre-Emmanuel Dauzat a explorée dan le livre qu ill a co-écrit avec vous Lenfer aussia ason orcheste. Mais peut-être pourriezvous, brièvement, nous expliquer comment cette « récupération » a bien pu avoir lieu ?

Tout à fait. Mais avant cela je voudrais préciser que la partition a été retrouvée par Francesco Lotoro, un chercheur et musicien italien. C'est a partir de ses travaux que j’ai été mis sur la piste de l'existence de cette œuvre et de son livret. Quant à une Jeanne d'Arc «juive »... C'est comme si, dans une certaine mesure, les Juifs étaient allés « récupérer», comme vous dites, la figure d’une femme guerrière, résistante, une liques ne méritaient plus tant que ça. Comme si, à ce moment-là, Jeanedít était plus utile aux Juifs qu'elle ne l'etait aux catholiques. Mais je préfère vous renvoyer au texte de Pierre-Emmanuel Dauzat qui, mieux que personne, a étudié les raisons historiques et culturelles profondes de cette «récupération».

La résistance à l'ennemi alors incarnée chez Viktor Ullmann à Theresienstadt par cette Jeanne d'Arc est exemplaire. Elle constitue l'essence même de la thématique et de la démarche inscrites au cœur de cet opéra dont le propos vise à se libérer de l'envahisseur et de la déportation. Pensez-vous qu'Ullmann ait élaboré cette œuvre en secret? Ou était-elle destinée à être jouée à Theresienstadt, comme la plupart de ses autres œuvres?

Il n’est pas toujours évident de savoir quelles œuvres ont été jouées, ou non, à Theresienstadt... Prenons, par exemple, le Kaiser von Atlantis. Les autorités juives ou allemandes du ghetto décidèrent d'interrompre les répétitions de l'opéra. On ne connait pas les raisons «officielles» de cette censure, mais entre l'hymne allemand qui y est caricaturé et le Kaiser qui ressemble un peu trop à un Führer, c’était impossible que cela passe... Mais s'agit-il d'une Jeanne d'Arc clandestine ? Difficile de le savoir. Comme il est aussi difficile d'imaginer ce que les autorités juives auraient fait d'une sainte catholique.

Mais pour moi, la vérité de cette Jeanne est ailleurs, si je puis dire... René Char a écrit un magnifique «portrait» de Jeanne d'Arc [Jeanne qu'on brûla verte] où il dit - évidemment, Char n'est pas croyant - qu'il se serait battu avec cette femme, à dit - evidemment, Char n'est pas croyant - qu'il se serait battu avec cette femme, à
ses côtés, parce qu'elle incarne à la fois l'insurgée et aussi l'extraordinaire mystique. Je pense qu'Ullmann aurait adoré cette vision...

Le texte du livret rend compte des circonstances qui menèrent Jeanne au bûcher. Outre la première thématique que nous venons d'explorer, celle de la "résistance », une seconde attire l'attention, qui concerne la «culpabilité » dont elle a fait preuve aux inquisiteurs après leur avoir annoncé vivre en état de grâce sous les auspices de Dieu. Mais cette déclaration, qui n'était qu' un leurre, lui devint rapidement insupportable à porter. Sa parole, en trahissant la vérité, l'avait elle-même blessée. Elle n'était plus que lexpression de sa propre faiblesse, due à sa peur de linquisition, de la question, de la torture et de la mort.

Elle dit, effectivement, que le plus grand péché qu'elle ait commis fut d’avoir menti à ces juges. C'est véritablement ce que l'on retrouve dans les minutes de son procès. On a les manuscrits. C'est la réalité historique. Naturellement, elle a eu peur de la mort et de la torture. Donc elle a commencé par mentir, puis elle est finalement allée jusqu’au bout de sa vérité, ce qui lui a coûté la vie.

II fallait donc - esprit de sacrifice insensé - qu'elle abandonne son corps à l'inquisition et donc à la mort pour que son âme, en retour, puisse être sauvée.

En somme, elle développe l'attitude inverse de celle d'un Faust offrant son âme au diable pour échapper à la mort, puisqu'elle l'offre à Dieu pour mourir en paix. Je ne sais diable pour échapper à la mort, puisqu'
pas si l'on peut établir un tel parallèle? 
Ce sentiment du "péché », d'une faute originelle à réparer, se trouve de fait aussi bien au cœur de la chrétienté que dans la religion juive. En imaginant le 30 mai 1431, Ullmann ne cherchait-il pas à accompagner le peuple martyr - les Juifs prisonniers et voués à lextermination - en rappelant solennellement leur grandeur dâme?

Je voudrais dire oui, mais je voudrais être certain que mon oui soit complet. On ne peut pas être dans son esprit. En revanche, il a fait une préface ${ }^{2}$, qui se trouve ne peut pas être dans son esprit. En revanche, il a fait une preface ${ }^{2}$, qui se trouve dans le livret et qui est assez mystérieuse, où il dit simplement qu'il était «partagé » entre un poème sur Jeanne d'Arc écrit par Schiller et la pièce de Bernard Shaw. Selon lui, Schiller noie, si je puis dire, l'Histoire par la Poésie contrairement à Shaw qui sacrifie tout pour la véracité historique. Ullmann dit que le personnage doit se situer entre Histoire et Poésie. Je crois donc qu'il a essayé dans son livret de faire une simple synthèse de ces deux «tempéraments».

Mais dans le cadre des camps, et ici du ghetto de Theresienstadt, on pourrait dire qu'il sagissaitinfine dexprimer une volonté dene pas fláchir, de resterdebout, dignes sas sion face à 'ennemi, pour mourir, certes, assassinés, mais intègres, dans les bras de Dieu?

Oui, il était sans doute trop tard pour être sauvé ailleurs que dans les bras de Dieu, comme vous dites.

Parlons donc à présent de la composition musicale de cet opéra. Des deux seules pages de la partition écrites par Ullmann, il n'était pas, semble-t-il, envisageable d'imaginer ce qu'il aurait pu concevoir pour l'achever.

Non, impossible. Ce serait comme espérer dessiner un visage à partir de l'esquisse d'une main.

Que pouviez-vous dès lors faire pour que soit connue et que vive cette œuvre inachevée? Comment, au départ du fragment existant, avez-vous pu nourrir et développer chevée? Comment, au départ du fragment existant, avez-vous pu nourrir et développer
l'œuvre? Vous avez choisi de vous positionner en tant que co-auteur, c'est-à-dire, plus l'œuvre? Vous avez choisi de vous positionner en tant que co-auteur, c'est-à-dire, plus exactement, de continuateur de l'œuvre. Alors, quelle est la part d'Ullmann et quelle
est la vôtre dans cette création devenue commune? Avez-vous tenu compte de l'œuvre est la vôtre dans cette création devenue commune? Avez-vous tenu compte de l'œuvre
complète d'Ullmann, en conservant son style, ou avez-vous développé une approche complète d'Ullmann, en conservant son style, ou avez-vous développé une approche (ceci pour rire entre nous) vous soufflant de l'achever au plus près de ce qu'elle aurait été si Ullmann avait eu le temps de l'achever?

Vous n'imaginez pas le nombre de personnes que j’ai croisées qui vous parlent de Jeanne d'Arc pour me dire qu'ils sont en communication avec elle. Le hasard fait que j'habite Rouen, la ville du bûcher. eproduction des deux pages existantes de la partition du

\section{II n'y a pas de hasard..}

et je peux vous dire que depuis la naissance de ce projet, je suis tombé sur des gens qui parlent avec Jeanne d'Arc comme je suis en train de vous parler. C'est-àdire que Jeanne d'Arc continue de fasciner d'une manière absolument hallucinante. Personnellement, je n’ai pas de lien direct avec Jeanne d'Arc. Voilà, je n’ai pas ce lien et je dois dire que je n'ai pas non plus un lien direct avec Ullmann, etj’aurais trouvéca assez curieux même indécent de m'imaginer qu'entre moi etUllmann, ily ait quelque assezc que mue chose qui se... non! Sil devait «y avoir quelque chose», en tout cas, c'est quelque part dans lincomming ingé parler à la place d'Ullmann, parler à travers lui, ou que lui parle par mabouche. Je ne suis pas un mystique halluciné. Je ne répudie pas toute forme de mysticisme ou de spiritualité, mais voilà, moi ce qui m’a semblé beau et terrible à la fois, le nœud de cette rencontre, c'est le silence abrupt de l'inachevé. Un silence d'une grande violence. Je me suis simplement demandé comment faire pour combler ça. À l'heure où je vous parle, le Dibbouk d'Ullmann ne m'habite pas... Ou alors, si c'est le cas, je ne m’en rends pas compte... Je suis resté moi, complètement moi-même. J'ai diffracté ces deux pages de musique d'Ullmann... Tout ça se passe quelque part entre du Ullmann à la manière d'Hélios Azoulay ou du Azoulay à la manière de Viktor Ullmann . Qui saurait dire qui commence ou Qui commence quoi ? Et si quelque chose est commencé ?... Mais arrêtez-moi, je vire mystique ! [rires]

Vous diffractez ?... à l'image d'un diamant, par exemple, qui diffracte les couleurs par l'incidence d'un rayon?

Mais peut-être, et c'est vrai que les éclats sur le mur... Enfin, absolument ! Je n’avais jamais... oui, la lumière, c'est ça.

\section{Y a-t-il eu une influence d'autres de ses opéras qui auraient...}

Pas du tout. Je connais très bien la musique d'Ullmann puisque je la joue régulièrement avec mon Ensemble. Mais je me suis concentré pour ne pas lécouter durant le temps de la composition. J'aurais trouvé cela tellement... Non cela ne peut pas marcher. Ce n’était pas un exercice de style. À de rarissimes exceptions, il n’y a rien de plus vulgaire et «inhabité» que ceux qui écrivent à la manière de.

D'accord, il s'agit donc d'un processus créateur à part entière de votre part.

Oui, je crois

Donc, d'un fragment musical, répété, varié, et en tenant compte du texte du livret, vous avez réussi à nous surprendre, certes, dans le bon sens du terme. Car en effet, l'œuvre d'Ullmann est mise en valeur et «vitw. Elle est, de notre point de vue - ceci dit 
sans ambages - belle et réussie, puissante, mais aussi, bien sûr, triste et terrible. Je suis sûr qu'Ullmann, s'il pouvait vous faire signe, vous manifesterait toute sa reconnaissance pour ce partage bien entendu néanmoins « forcé ».

Merci. [rires]

Votre développement de l'œuvre s'organisant à partir des deux pages existantes de la partition et sur le livret achevé d'Ullmann, comment avez-vous procédé? Les instruments avaient-ils été choisis par Ullmann?

Sur le manuscrit, il y a quelques instruments désignés, notamment un hautbois et trois trompettes. Le reste étant sans doute les instruments à cordes. Quant à moi, je voulais quelque chose de souple. Je ne voulais pas écrire une pièce pour orchestre pour plusieurs raisons. C'est que quand vous écrivez une pièce pour orchestre et qu'il y a une chanteuse et un récitant, vous vous condamnez à écrire une sorte d'oratorio, et «l'ambiance messe » ne m’intéresse pas du tout... Je ne voulais pas non plus faire un opéra, parce que je n'avais pas envie d'être encombré par un metteur en scène. Je voulais que cela soit une œuvre que l'on puisse partager de manière trè simple. Le quatuor à cordes m'a semblé de manière tout à fait évidente la bonne simple. Le lutuor à cordes ma semblé de manière tout a fait evidente la bonne formule, la plus pure, la plus "noir et blanc", pour redonner vie à cette œuvre, pour lui donner vie...

Marielle Rubens porte superbement la voix de Jeanne, tandis que vous interprétent a coup, en vous écoutant, en entendant la voix et le chant, c'est tout le spectacle de l'opéra qui tend à se manifester sous nos yeux. Pensez-vous, malgré vos réserves, qu'il soit possible d'en réaliser également une mise en scène?

Il ne faudrait pas le dire, maisj’ai rarement rencontré un metteur en scène dont le travail me passionne. Donc si un jour j'en rencontre un que je trouve honnête, je travaillerai avec lui très volontiers. Mais souvent pour moi la mise en scène... je vais être très sévère, ce n'est pas grave...?

\section{Non, non... [rires]}

Trop tard! Très souvent, les metteurs en scène marchent sur le crâne d'une œuvre avec leurs chaussures à crampons... Et pensent, parce que le texte gueule, qu'ils le font parler...

Finalement, il vaut peut-être mieux que l’opéra soit évoqué comme vous le dites justement... Que le public ferme les yeux et voie... Parce qu'il vaut mieux voir que se laisser montrer, non ?... Et puis un chœur, un chef d'orchestre ? Convaincre tout le monde? Non, je n'ai pas du tout envie de cela. Mais présentez-moi un génie et je retire tout ce que jai dit! [rires]
D'accord, c'est très clair.

Pierre-Emmanuel Dauzat, co-auteur de votre livre L'enfer aussi a son orchestre, a écrit un texte dans le livret qui accompagne votre $C D$. Il y fait notamment référence au mythe d'Orphée et Eurydice, qui fut interprété par tant de compositeurs célèbres à travers l'histoire de la musique. II apparaît effectivement que dans le contexte des ghettos et des camps, dont celui de Theresienstadt qui nous occupe ici, les déportés savaient qu'ils seraient, un jour ou l'autre, menés à « pénétrer » les territoires d'outre-tombe, exécutés dans les chambres à gaz d'Auschwitz-Birkenau. Jeanne, morte en plein acte de rédemption, incarnant et représentant ici par
Janter extension la communauté juive, peut-elle être comparée à Eurydice? En s engageant même dans notre cas bien malgré lui, dans les territoires de lihades, lespoir de la retrouver - de retrouver son peuple - et d'en revenir, est sans doute l'ultime message d'espoir et de résistance «philosophique » délivré par Ullmann avant sa propre disparition. Comment une telle œuvre a-t-elle pu être, tout simplement, écrite dans ce contexte ? On voit que la création reste essentielle à la vie comme une émanation qui lui est consubstantielle.

Tout à fait. Mais je ne crois pas que nous disposions du temps nécessaire pour éclairer un tel mystère. Et quand bien même nous aurions la vie devant nous, un mystère comme celui-là, même éclairé, ne peut être qu'effleuré... Cela me rappelle une interview que jai donnée récemment pour la telévision israélienne, par téléphone, en direct, où on ma dit: «Pourquoi y avait-il de la musique et de l'art dans les camps de concentration ? En deux ou trois phrases...» Mais ce n'est pas une question ça, c'est un vertige !

En fait, lorsqu'on apprend qu'il y avait de la musique dans les camps, on est tout d'abord étourdi de surprise. Mais plus on réfléchit à cette présence, plus on se rend compte que la musique est présente à tous les moments de notre existence. Donc pourquoi pas là aussi ? Et cela nous enseigne peut-être aussi qu'en fouillant dans la poche de la catastrophe, on y trouve parfois un miracle. Je crois, plus qu'ailleurs, plus que partout ailleurs, que ces musiques surgies là-bas, sont des miracles. Et le . Et le fait qu il y ait tant douve d' Ullmann est un miracle d'une telle densité ! Tellement inattendu, inimaginable, dingue... Jeanne d'rc dans les camps ! Cela semble si inpensable... Elle ne devrait rien avoir à faire là, et pourtant si ! Souvenez-vous de René Char... Jeanne d’Arc, l'insurgée et la mystique. C'est parfois bien plus utile qu'une sainte [rires].

En fait je voudrais dire, et c'est important pour moi de le dire, que je ne me suis pas engagé dans cette œuvre-là en pensant qu'elle puisse être l'allégorie, la métaphore, le symbole de quelque chose. Le génie de cette Jeanne d'Ullmann, même inachevée, est d'exister, uniquement d'exister. Il n'y a rien d'autre à dire. Elle est pour moi, simplement, une présence impensable. Et il y avait déjà tellement dans cette présence que je n’ai pas commencé, à aucun moment, à l'analyser ou à l’interpréter. 


\section{C'est ce que je cherchais un petit peu à faire là.}

C'est sans doute inévitable et peut-être est-ce, en partie, le rôle de ceux qui l'écoutent?

Oui, c'est ça, voilà, parce que l'œuvre agit un peu, tout de même, comme une lumière qui pompe, puise et reflète l'énergie qui se trouve autour d'elle pour la refléter, pour en réfléchir sa propre substance et la partager, me semble-t-il, avec l'entourage?

Cela a l'air, peut-être, d'être une manière de parler, mais je crois, et c'est curieux, que j’ai essayé deêtre le plus, le mot me vient à la bouche pour la première fois, mais si je puis dire, le plus «détaché» possible. Et je crois que c'est dans ce détachement «satellitaire» que je me suis trouvé le plus au cœur des choses. Il me semble, il me semble..

Oui, mais n'est-ce pas parce qu'il fallait que vous trouviez une voie créative pour développer une œuvre qui avait déjà été initiée, et en même temps, comme vous l'avez très bien expliqué, ne pas essayer de plagier d'une manière ou d'une autre en imaginant

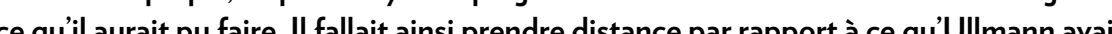
fait et rester tout de même dans le cadre. Il y avait là une juste mesure à trouver.

Exactement, à mon avis, plus juste est la distance plus grande est la proximité, Je crois d'ailleurs que c'est ce qu'incarne la poésie, plus que tout. La poésie, à mon sens, l'expression poétique, littéraire, c'est précisément la distance à laquelle on se tient des mots quand on écrit... Il y a entre les mots et leur sens communémen admis une distance... C'est là qu'il faut travailler... Et c'est de la juste distance avec les mots que nait l'éclaircie de ce que vous voulez leur faire dire, ou de ce qu'ils veulent bien vous dire... Car ce sont souvent les mots qui nous emploient plus que nous en sommes les employeurs . Et pour moi tout ca c'est très, si je puis dire, chagallien! C'est-à-dire qu'il faut faire voler les vaches, faire jouer les violonistes sur les toits

Et bien merci beaucoup Hélios. Donc, on ne peut que s'en rendre compte, et pour achever notre conversation, que cette œuvre, Le 30 mai 1431, va rapidement s'avére primordiale et essentielle parmi celles d'Ullmann, et parmi vos propres compositions.

Je l'espère de tout cœur, merci.

\section{SAUVÉE DES CENDRES}

Hélios Azoulay et l'Ensemble de Musique Incidentale

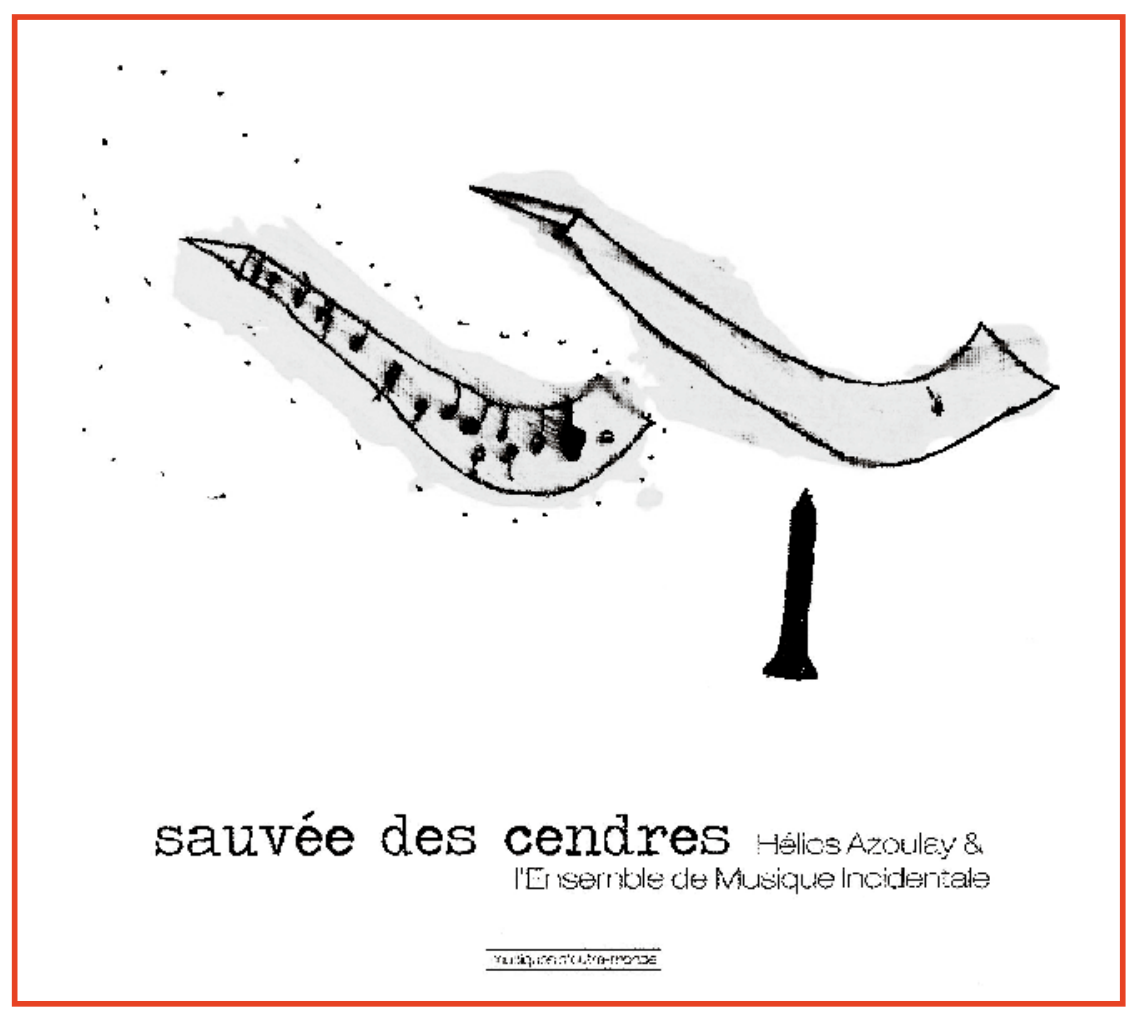

\section{HÉLIOS AZOULAY}

direction musicale

'Ensemble de Musique Incidentale

Enregistré au studio ACCES DIGITAL, Rouen (octobre, novembre 2016) Ingénieur du son et mixage : François Casays

() Illustrations : inocsence complète, Caspar (2015) - Infographie : Boris Ivanenko Traduction de Yallemand [2-7] : Henri Prusak \& Hélios Azoulay [8] : Marielle Rubens Traduction du polonais [10] : Henri Prusak

Le livret de Viktor Ullmann Le 30 mai 1431, est publié intégralement dans

(c) Ensemble de Musique Incidentale - www.heliosazoulay.com 


\section{ALEKSANDER VOLKOVISKI Shtiler Shtiler}

5'49"

Hélios Azoulay piano

\section{[2 - 7] \\ VIKTOR ULLMANN \& HÉLIOS AZOULAY HÉLIOS AZOULAY \& VIKTOR ULLMANN \\ Le 30 mai 1431}

[2] Acte I, scène 1 - 4'19"'

[3] Acte I, scène 2 - 3'29"

[4] Acte I, scène 3 - 3'03"

[5] Acte II, scène 1 - 3'59"

[6] Acte II, scène 2 - 6'29"

[7] Acte II, scène 3 - 8'01"

Marielle Rubens chant

Hélios Azoulay suprême Clairon, récitant

Pablo Schatzman violon

Teona Kharadze violon

Baptiste Vay alto

Dimitri Maslennikov violoncelle

\section{[8] \\ ILSE WEBER \\ Und der Regen rinnt 2'29"}

Und der Regen rinnt, und der Regen rinnt, ich denk im Dunkeln an dich, mein Kind. Hoch sind die Berge und tief ist das Meer, mein Herz ist müd und sehnsuchtsschwer. Und der Regen rinnt, und der Regen rinnt, warum bist du so fern, mein Kind?

Und der Regen rinnt, und der Regen rinnt, Gott selbst hat uns getrennt, mein Kind. Du sollst nicht Leid und Elend sehn, sollst nicht auf steinigen Gassen gehn.

Und der Regen rinnt, und der Regen rinnt, hast du mich nicht vergessen, Kind?

Marielle Rubens chant

Hélios Azoulay piano
[9]

\section{HÉLIOS AZOULAY}

La Rêverie de Mengele, ורכזו ומש חמי

8'25"

Pablo Schatzman violon

Teona Kharadze violon

Baptiste Vay alto

Dimitri Maslennikov violoncelle

\section{AARON LIEBESKIND}

Kołysanka dla synka w krematorium 4'12"

Krematorium czarne, gtuche

Bramy piekiet, trupów stos

Śliskie, sztywne ciata wloke

Osiwiatem w jedna noc

Oto synek leży, synek mój

Mate piastki $w$ usta wgryzł

Jakżeż ciebie w ogień wrzucę tu!

Ztote wtoski śliczne twe

Lulaj, lulaj... synku mój

Lulaj, lulaj... synku mój

Lulaj, lulaj... synku mój

Synku mój

Podte stońce, czemu milczysz?

Wszak widziatem wszystko tu

Gtowkejego roztrzaskali

O kamienny, zimny mur

Patrza w niebo ciche oczka twe

I zastygte krzycza tzy

Synku! Wszędzie, wszędzie twoja krew!

A przeżyteślatka... trzy

Lulaj, lulaj... synku mój

Lulaj, lulaj... synku mój

Lulaj, lulaj... synku mój

Synku mój

Marielle Rubens chant 